\title{
Ten Digital Health Tensions Driving Innovation in MedTech
}

Christopher L. Wasden, EdD

The biggest failure in healthcare is the tendency to overlook the fact that the consumer is the patient. Consequently, all stakeholders (e.g., governments, private payers, medtech and pharma companies, and providers) almost always ignore consumer-oriented value propositions. Instead, they focus on creating and delivering their value propositions with one another. This has resulted in healthcare systems around the world failing to deliver on the triple aim of affordable, accessible, and effective healthcare-with the most pronounced failures occurring in the United States.

Four digital technologies I call SMAC (Social, Mobile, Analytic, and Cloud) are now putting the consumer at the center of healthcare and beginning to radically disrupt healthcare as they already have retail, travel, and media. This disruption is now creating powerful tensions that are creating the "end of healthcare and medtech as we know it" and ushering in the digital health revolution.

\section{So what's the problem?}

Medtech firms have not yet figured out how to harness the 10 tensions created by these new digital health technologies to validate, package, and promote their solutions as effectively as drug companies do to drive mass adoption.

1. Money Tension: Clinicians and health systems will only adopt digital health if they can make more money; yet the primary value of digital health is to improve the triple aim: better outcomes, greater access, and affordable care (lower costs). Digital health has the power to decrease costs by $50 \%$ or more.

2. Practice Tension: Digital health must drive creative destruction of medicine and transform the practice of healthcare to one that uses fewer clinicians. Pharma and medtech are leading this transformation. Digital health eliminates all physician and nurse shortages around the world.

3. Engagement Tension: Consumers must be more engaged in making healthy decisions but less engaged in the health system. Digital health provides platforms that enable this. Consumers 
want the same type and quality of digital engagement in healthcare they get in all other aspects of their lives.

4. Adoption Tension: Those needing digital healthcare the least are most likely and willing to adopt it the fastest, while those needing it the most will be slow adopters. Early adopters provide valuable tests cases to drive innovation. We must learn from the successes of companies like Fitbit $i^{\circledR}$ in order to achieve the same adoption in other aspects of health.

5. Technology Tension: We have an over abundance of technologies but an inability or unwillingness by clinicians to use them. Technology adoption must become as simple as writing a prescription and require no more thinking and effort from clinicians. We must create a digital health app formulary to make adoption by clinicians simple and easy.

6. Satisfaction Tension: Nearly every study and survey on digital health shows $85 \%+$ consumer satisfaction due to significantly better outcomes (e.g., shorter hospitalization, fewer admissions and readmissions, happy people). Consumers must become outspoken revolutionaries demanding digital health solutions. We must eliminate paternalism in healthcare and put the consumer in charge.

7. Feedback Tension: Currently, there is virtually no feedback loop in healthcare for the vast majority of consumers. Digital health enables real time feedback, but not by the human physician. We must create feedback loops that don't require the physician.

8. Utilization Tension: Nearly all studies of digital health show $20 \%$ to $40 \%$ reductions in health system utilization, yet health systems in developed countries can't figure out how to turn that into economic value. Emerging markets and consumer-driven systems are creating new digital health systems to leapfrog developed markets. We must penalize waste and incentivize value so we are not afraid to keep people out of the healthcare system.

9. Validation Tension: Despite a plethora of pilots and studies of digital health applications and use cases, we lack a systemic way to evaluate and validate those that work and their level of effectiveness. We need social and crowdsourced mechanisms to rapidly test and share digital health solutions. Peer-reviewed articles that take three to five years to produce will never keep up with the pace of digital health innovation and can't be the standard for validation. [Editor's note: the TMT review time is one to two months from submission to publication.] 
10. Behavior Change Tension: Digital health's value only really accrues by changing consumer and clinician behaviors, yet clinicians have insufficient training in behavioral psychology, economics, and change. And this is compounded by insufficient time for useful patient/physician discussion. Digital health vendors must incorporate behavior change science into their solutions just as Google, Amazon, Facebook, and Apple do in digital technology solutions. We must embed behavior change into gamified apps and devices so we don't require clinicians to figure out how to change behavior on a one-by-one basis.

Those medtech organizations that harness these 10 tensions will improve costs, efficacy, safety, and outcomes to deliver successful blockbusters digital health technologies through new business models to consumers in the market.

Tags: accessible, affordable, consumer, cost, effective, efficacy, health care, healthcare, outcome, safety,

Christopher L. Wasden, EdD, is Professor of Innovation \& Executive Director of the Sorenson Center for Discovery and Innovation at University of Utah. This article is based on the author's book, Wasden C, Wasden M. Tension: The Energy of Innovation. How Harnessing Tension Accelerates and Fuels Your Creative Genius. Midway (UT): Scipio Press; 2015. ISBN 978-1-944465-01-8 (hardcover). 